\title{
Clusterin: a key player in cancer chemoresistance and its inhibition
}

This article was published in the following Dove Press journal:

OncoTargets and Therapy

20 March 2014

Number of times this article has been viewed

\section{Tomas Koltai}

Gerencia de Efectores Sanitarios Propios, Instituto Nacional de la Seguridad Social para Jubilados y Pensionados, Buenos Aires, República Argentina
Correspondence: Tomas Koltai Gerencia de Efectores Sanitarios Propios. Instituto Nacional de la Seguridad Social para Jubilados y Pensionados, Buenos Aires, República Argentina Email tkoltai@pami.org.ar
Abstract: Clusterin is a heterodimeric disulfide-linked glycoprotein (449 amino acids) isolated in the rat prostate after castration. It is widely distributed in different tissues and highly conserved in species. There are two isoforms ( 1 and 2 ) with antagonistic actions regarding apoptosis. Clusterin is implicated in a number of biological processes, including lipid transport, membrane recycling, cell adhesion, programmed cell death, and complement cascade, representing a truly multifunctional protein. Isoform 2 is overexpressed under cellular stress conditions and protects cells from apoptosis by impeding Bax actions on the mitochondrial membrane and exerts other protumor activities, like phosphatidylinositol 3-kinase/protein kinase B pathway activation, modulation of extracellular signal-regulated kinase 1/2 signaling and matrix metallopeptidase- 9 expression, increased angiogenesis, modulation of the nuclear factor kappa B pathway, among others. Its overexpression should be considered as a nonspecific cellular response to a wide variety of tissue insults like cytotoxic chemotherapy, radiation, excess of free oxygen radicals, androgen or estrogen deprivation, etc. A review of the recent literature strongly suggests potential roles for custirsen in particular, and proapoptosis treatments in general, as novel modalities in cancer management. Inhibition of clusterin is known to increase the cytotoxic effects of chemotherapeutic agents, and custirsen, a second-generation antisense oligonucleotide that blocks clusterin, is being tested in a Phase III clinical trial after successful results were achieved in Phase II studies. A major issue in cancer evolution that remains unanswered is whether clusterin represents a driving force of tumorigenesis or a late phenomenon after chemotherapy. This review presents preclinical data that encourages trials in various types of cancer other than advanced castration-resistance prostate cancer and discusses briefly the appropriate timing for clusterin inhibition in the clinical context.

Keywords: custirsen, apoptosis, renal cell carcinoma, triple negative breast cancer, castrationresistance prostate cancer

\section{Introduction}

Castration in rats leads to involution of the ventral prostate, because this organ requires adequate concentration of androgens. One of the mechanisms involved in prostate involution is cell death. Castration elicits the appearance of several proteins in the prostate whose functions were unknown at the time. In 1989, a messenger (m)-RNA coding for sulfated glycoprotein-2 was identified in the prostate of castrated rats, peaking between days 3-4 after castration. ${ }^{1}$

Up to 1995, there was the concept that sulfated glycoprotein-2 (also dubbed apolipoprotein J or testosterone-repressed message-2), best known now as clusterin (CLU), was a protein associated with reduction in programmed cell death (apoptosis) in the prostate, but its exact role remained uncertain. This role was clarified by pioneering 
studies performed by Sensibar et $\mathrm{al}^{2}$ working on LNCap cells, which are androgen-sensitive human prostatic cancer cells obtained from a human lymph node metastasis.

This research showed that CLU was associated with protection against induced cell death, reducing the cytotoxic effect of tumor necrosis factor (TNF)- $\alpha$ in LNCap cells. The overexpression of CLU, resulting in resistance to TNF- $\alpha$-induced apoptosis, was not described prior to this research, which included the finding that there was a correlation between the expressed CLU and degree of resistance to TNF- $\alpha$-induced apoptosis. The authors proposed the hypothesis that CLU overexpression can prevent cell death, whereas its depletion is responsible for the onset of cell death.

These data were later confirmed and amplified in a study showing that CLU knockdown in human cancer cells induced significant reduction of cellular growth and higher rates of spontaneous endogenous apoptosis. ${ }^{3}$

\section{CLU gene}

The $C L U$ gene is a well conserved gene during evolution, located in humans in chromosome 8 (8p21-p12) and it is expressed during tissue regression in many organs. ${ }^{4}$ Its overexpression should be considered as a nonspecific cellular response to a wide variety of tissue insults. ${ }^{5}$

$C L U$ is a single nine-exon gene expressing three protein forms with different subcellular localizations and diverse biological functions. ${ }^{6}$

\section{Clusterin isoforms and structure}

However, things are not that simple regarding CLU, because we now know that the $C L U$ gene has nine exons and there are at least three isoforms of clusterin: isoform 1, isoform 2, and isoform 11036. Exon 1 has three portions: 1a, 1c, and 1b.

Isoform 1 (nCLU, nuclear clusterin) is the product of the nine exons but contains only part 1 a of exon 1 , whereas isoform 2 (sCLU, secretory clusterin), contains the product of part $1 \mathrm{~b}$ of exon 1 and isoform 11036 (cCLU, cytoplasmic CLU) contains part 1c of exon 1 (Figure 1). ${ }^{6,8}$ sCLU and nCLU are originated by the $C L U$ gene through alternative splicing. nCLU is a slightly longer mRNA and with a completely different function: binding to $\mathrm{Ku} 70$ and promoting cell death by freeing Bax.

In summary, the $C L U$ gene produces through alternative splicing three different proteins, two of which are sCLU, which prevents cell death, and nCLU, which promotes cell death. One CLU form is secreted (sCLU), whereas the other forms have a cytoplasmic (cCLU) or nuclear (nCLU) localization.

A unified nomenclature has been proposed. ${ }^{9}$ sCLU is reserved for the main gene transcript identified from the $C L U$ gene locus. It has a predicted molecular mass of $50.1 \mathrm{kDa}$, which is detected as a $60 \mathrm{kDa}$ glycosylated presecretory form of sCLU and is referred to as psCLU. psCLU is translated from the mRNA, including the nine gene exons. Once it forms the mature, secreted heterodimeric 75-80 kDa protein, it should be referred to as sCLU. Thus, mature sCLU is a heterodimeric disulfide-linked

A

CLU gene

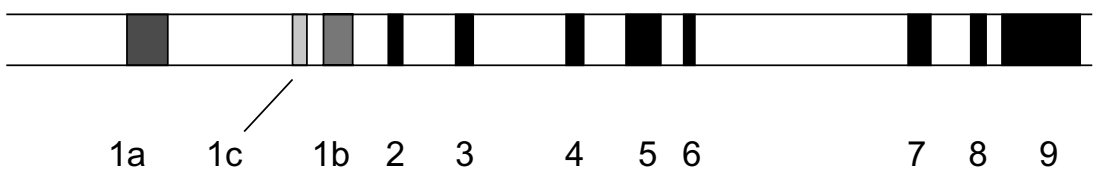

B

CLU transcript

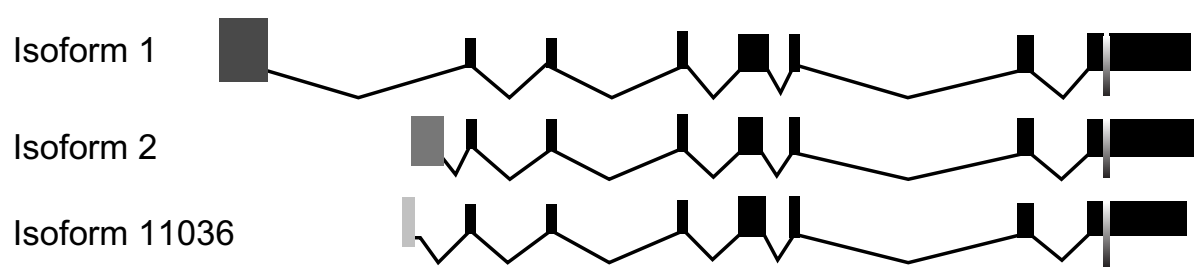

Figure I Structure of CLU gene and transcription products. (A) CLU gene maps on chromosome 8. The position of exons and introns on the gene is shown. Black bands: exons, white bands: introns. (B) Exonic structure of CLU mRNA variants. Dark grey exon, exon Ia of isoform I; medium grey exon, exon Ib of isoform 2; light grey exon, exon Ic of isoform II036.

Note: Reproduced with permission from BioScientifica Ltd. Copyright ( 2010 , Society for Endocrinology. Rizzi F, Bettuzzi S. The clusterin paradigm in prostate and breast carcinogenesis. Endocr Relat Cancer. 2010;17(I):RI-RI7.6

Abbreviation: CLU, clusterin. 
glycoprotein (449 amino acids). It is composed of two subunits $(\alpha$ and $\beta$ ) resulting from the cleavage of psCLU. sCLU has a cytoprotective and chaperone role. sCLU was described as an inhibitor of the cytolytic reaction of terminal complement proteins.

The existence of the two isoforms may explain how progression toward high-grade and metastatic carcinoma leads to a shift from nCLU to sCLU expression, and that the role of $C L U$ for tumor growth may be related to a pattern shift in its isoform production. ${ }^{10}$

Essabbani et $\mathrm{al}^{8}$ showed that increasing the nCLU:sCLU expression ratio by using an "on demand alternative splicing" strategy successfully increased sensitivity to radiotherapy and chemotherapy of prostate cancer cells, and this research showed clearly the different functions of these two isoforms.

Isoform 1 and isoform 2 are both found in prostate cancer cell lines but are differentially regulated by androgens: whereas isoform 2 (sCLU) is upregulated, isoform 1 (nCLU) is downregulated. ${ }^{11}$

\section{Clusterin actions}

CLU protects cells from various stressors that may induce apoptosis, like cytotoxic chemotherapy and androgen or estrogen deprivation. The cytoprotective role of CLU can be explained by its chaperone-like activity. ${ }^{12} \mathrm{CLU}$ expression is low in most normal cells and is strongly stimulated by various stressors. ${ }^{5,13}$

How does CLU prevent cell death? The answer came in 2009: sCLU stabilizes the union between Klu70 and the apoptotic protein Bax, thereby preventing Bax from joining the mitochondrial outer membrane and exerting its proapoptotic activity, so that elevated sCLU levels may enhance tumorigenesis by interfering with Bax proapoptotic activities (Figure 2). ${ }^{14}$

Depletion of sCLU triggers two types of action: 1) p53 dependent; and 2) p53 independent (Figure 3). ${ }^{14-18} \mathrm{sCLU}$ depletion activates p53 and alters the ratio of proapoptotic to antiapoptotic $\mathrm{Bcl}-2$ protein family members, triggering mitochondrial dysfunction and apoptosis. However, Bcl-2 suppression is a p53-independent effect, such that knockdown of p53 only minimally suppressed cell death, but a pan-caspase inhibitor completely avoided cell death. Another mechanism involved in sCLU prosurvival activity is the upregulation of the

Bax-Ku70 complex + sCLU $\longrightarrow$ Bax-Ku70-sCLU complex: no apoptosis* Bax-Ku70 complex + sCLU depletion $\longrightarrow$ Free Bax: apoptosis

Figure 2 Main mechanism of sCLU action.

Note: *Due to its capacity to bind Ku70, CLU is also dubbed Ku70 binding protein-I.

Abbreviation: $\mathrm{sCLU}$, secretory clusterin. phosphatidylinositol 3- kinase (PI3K)/protein kinase B (AKT) pathway ${ }^{15}$ and insulin-like growth factor (IGF)-1 activates the PI3K/AKT pathway through upregulation of sCLU. ${ }^{16}$

Besides its important role in apoptosis, sCLU has other actions related to tumor behavior. For example, in clear cell renal carcinoma (CCRC) sCLU regulates aggressive behavior by modulating extracellular signal-regulated kinase (ERK) $1 / 2$ signaling and matrix metallopeptidase (MMP)-9 expression. ${ }^{17}$ Knockdown of sCLU in cells showed reduced migration and invasion in vitro, as well as decreased metastatic potential in experimental metastasis.

Overexpression of CLU in epithelial ovarian cancer appears to be correlated with increased tumor angiogenesis, consistent with the established role of $C L U$ as an oncogene in the biology of ovarian cancer. ${ }^{18}$ Also, sCLU increased chemoresistance in ovarian cancer cells. ${ }^{19}$

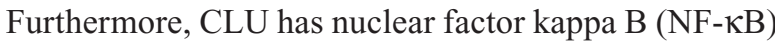
regulatory actions. ${ }^{20}$ In prostate cancer cells, Zoubeidi et al ${ }^{21}$ discovered sCLU's ability to regulate ubiquitination and degradation rates of COMM domain-containing protein 1 and inhibitor of kappa B to enhance NF- $\mathrm{KB}$ nuclear translocation and transcriptional activity. Accordingly, sCLU knockdown stabilizes COMM domain-containing protein 1 and inhibitor of kappa B, sequesters NF- $\kappa B$ in the cytoplasm, and decreases transcription of many NF- $\kappa B$ regulated genes.

CLU is a protein widely distributed among cells, but certain situations like cellular stress, like chemotherapy, radiotherapy, or androgen depletion in prostate trigger CLU overexpression. CLU expression is under genetic and epigenetic control, the latter via methylation of its promoter. ${ }^{22}$

Many strategies used to kill cancer cells induce stress responses that activate survival pathways, including overexpression of sCLU, to promote emergence of a treatment resistant phenotype.

There is a strong association between CLU and glucoseregulated protein, according to Wang et al. ${ }^{23}$ Apparently CLU protects cells from endoplasmic reticulum stress-induced apoptosis, at least partially through interacting with glucoseregulated protein.

sCLU is a stress-activated cytoprotective chaperone upregulated by many varied anticancer therapies to confer treatment resistance when overexpressed. ${ }^{24}$ Using size exclusion chromatography and fluorescently labeled paclitaxel, Park et $\mathrm{a}^{25}$ demonstrated that clusterin binds to paclitaxel. These authors also showed that high levels of clusterin expression increased paclitaxel resistance in ovarian cancer cells by physically binding to paclitaxel, which may prevent paclitaxel from interacting with microtubules to induce apoptosis. 


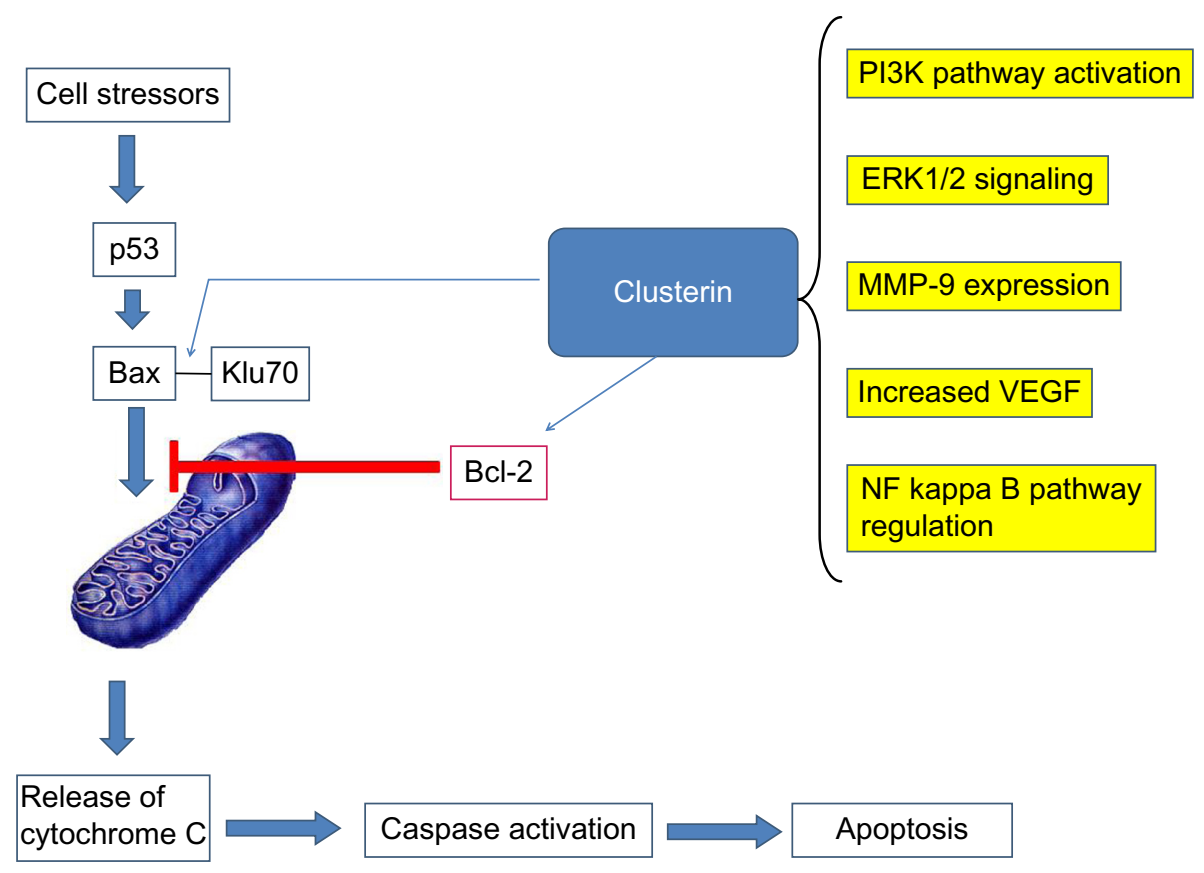

Figure 3 Mechanisms of clusterin action.

Notes: On the left side, two antiapoptotic mechanisms are described (p53-dependent and $\mathrm{p} 53$-independent). On the right side, other protumor mechanisms are not directly related with apoptosis.

Abbreviations: ERK, extracellular signal-regulated kinase; MMP, matrix metallopeptidase; NF, nuclear factor; PI3K, phosphatidylinositol 3- kinase; VEGF, vascular endothelial growth factor.

CLU expression is downregulated in human testicular seminoma, which is a highly sensitive tumor to radiotherapy and chemotherapy. ${ }^{26}$ The authors postulate that CLU expression in human testicular seminoma differs from CLU expression in other tumors and think that this fact may be the cause of the treatable characteristics of testicular seminoma.

There is no doubt nowadays that CLU has an important role in tumorigenesis and progression of human cancers, ${ }^{8}$ therefore it is a valid target for new cancer treatment modalities. Targeting the apoptosis pathway ${ }^{27}$ is a new and promising therapy.

\section{Clusterin regulation}

sCLU is overexpressed when stressor conditions are present in the cell, but at least in the case of ionizing radiation as a stressor condition, a regulatory pathway for CLU overexpression was identified: activation of ataxia telangiectasia-mutated kinase by endogenous or exogenous forms of DNA damage was required to relieve basal repression of IGF-1 transcription by the p53/NF-YA complex, leading to sCLU expression. ${ }^{28}$ Cells exposed to DNA-damaging agents had elevated IGF-1 expression, resulting in activation of IGF-1R signaling and sCLU induction. Overexpression of H-Ras caused repression of CLU expression. ${ }^{29}$ These authors support the concept that apoptotic processes, not oncogenic mutations, are responsible for increased clusterin expression in tumors. p53 exerts a role in the transcriptional repression of sCLU. Loss of functional p53 in HCT116:p53(-/-) cells augmented CLU de novo synthesis after IR exposure. Repression of sCLU protein levels by $\mathrm{p} 53$ may be important for the cascade of p53-mediated events leading to cell death after IR or other cytotoxic agent exposure. ${ }^{30}$

\section{Clusterin inhibition}

Custirsen (OGX-011; OncoGeneX Pharmaceuticals, Inc., Bothell, WA, USA) is a second-generation antisense oligonucleotide designed to block mRNA sCLU, producing depletion of the protein sCLU and improving clinical results in advanced prostatic cancer resistant to castration. Custirsen is currently in Phase III clinical trials. ${ }^{31}$ Even so, there is evidence that custirsen could be helpful in other kinds of tumors besides prostate cancer, such as CCRC,,${ }^{17,32-34}$ breast cancer, ${ }^{35-38}$ lung cancer, ${ }^{39}$ and cancer of the ovary, ${ }^{18,40}$ to mention a few.

\section{The reasons for custirsen development}

Custirsen binds to exon 1 of mRNA $s C L U$, blocking its translation. The development of this novel drug was due to findings like:

1. Renal carcinoma cells overexpressing sCLU became more resistant to apoptosis. ${ }^{41}$ 
2. Oligonucleotides that target sCLU increase sensitivity to radiation in prostate cancer cells. ${ }^{42}$

3. Oligonucleotides that target sCLU increase sensitivity to chemotherapeutic agents. $^{43}$

\section{Possible custirsen activity in various tumors}

Custirsen has been found to increase cytotoxicity when associated with docetaxel in prostate carcinoma cells, ${ }^{31}$ with sorafenib in CCRC, ${ }^{33}$ with cisplatin in CCRC, ${ }^{34}$ with gemcitabine and platinum compounds in lung cancer cells, ${ }^{39}$ and with paclitaxel. ${ }^{44}$

\section{CLU and prostate cancer}

It has been extensively investigated and there are many publications and reviews on this subject. Custirsen is being tested in phase III clinical trials as treatment for advanced prostate cancer resistant to castration in association with docetaxel. For a detailed analysis of CLU in prostate cancer, see the review by Higano. ${ }^{31}$

\section{CLU and breast cancer}

Table 1 summarizes the findings from various studies of breast cancer discussed below. sCLU overexpression was associated with resistance to preoperative neoadjuvant chemotherapy in primary breast cancer. ${ }^{35}$

In breast cancer, the situation may be paralleled to that of prostate: the research by Wang et $\mathrm{al}^{45}$ suggests that sCLU confers to breast cancer cells resistance to TNF-

Table I Summary of findings in breast cancer

\begin{tabular}{|c|c|}
\hline Reference & Results \\
\hline 35 & $\begin{array}{l}\text { sCLU overexpression is associated with resistance to } \\
\text { preoperative neoadjuvant chemotherapy }\end{array}$ \\
\hline 45 & $\begin{array}{l}\text { sCLU confers breast cancer cells resistance to TNF- } \alpha \\
\text { apoptosis }\end{array}$ \\
\hline 36 & $\begin{array}{l}\text { sCLU silencing significantly inhibited the proliferation of } \\
\text { MDA-MB- } 23 \text { I cells, and decreased dramatically invasion } \\
\text { and migration }\end{array}$ \\
\hline 37 & $\begin{array}{l}\text { sCLU knockdown in MDA- } 23 \text { I cells reduced cell } \\
\text { migration and invasion and decreased metastatic potential }\end{array}$ \\
\hline 46 & $\begin{array}{l}\text { Overexpression of sCLU is seen in higher percentage in } \\
\text { triple negative breast cancer }\end{array}$ \\
\hline 47 & $\begin{array}{l}\text { sCLU expression is associated with negative estrogen } \\
\text { and progesterone receptor status }\end{array}$ \\
\hline 45 & Clusterin inhibition enhanced paclitaxel sensitivity \\
\hline 48 & $\begin{array}{l}\text { Custirsen did not show advantages when associated with } \\
\text { chemotherapy of breast cancer }\end{array}$ \\
\hline 38 & $\begin{array}{l}\text { Clusterin overexpression enhances metastatic } \\
\text { progression }\end{array}$ \\
\hline
\end{tabular}

Abbreviations: $\mathrm{sCLU}$, secretory clusterin; TNF, tumor necrosis factor. $\alpha$-induced apoptosis through NF- $\mathrm{\kappa B}$ activation and Bcl-2 overexpression.

Niu et $\mathrm{al}^{36}$ investigated the effects of $s C L U$ inhibition with small interfering RNAs (siRNAs) on cell motility, invasion, and growth in vitro and in vivo in breast cancer cells. MDAMB-231 cells were transfected with pSuper-siRNA/sCLU. The results showed that $S C L U$ silencing significantly inhibited the proliferation of MDA-MB-231 cells. The invasion and migration ability were also dramatically decreased. They also demonstrated that $s C L U$ silencing could increase the apoptosis rate of cells, resulting in the inhibition of cell growth. The effects of $s C L U$ silencing on tumor growth and metastatic progression in an orthotopic breast cancer model showed that orthotopic primary tumors derived from MDAMB-231/pSuper sCLU siRNA cells grew significantly slower than tumors derived from parental MDA-MB-231 or MDAMB-231/pSuper scramble siRNA cells, and metastasized less to the lungs. These data suggest that sCLU plays a significant role in tumor growth and metastatic progression in certain breast cancer cell lines. The authors consider that knocking down $s C L U$ gene expression may provide a valuable method for breast cancer therapy.

sCLU knockdown in the MDA-231 breast cancer cell line reduced cell migration and invasion in vitro and decreased metastatic potential. ${ }^{37}$

Triple-negative breast cancer patients were positive for sCLU in a remarkably higher percentage than were patients who were not triple negative, based on immunohistochemical analysis, and sCLU expression. In this triple negative subtype, a larger tumor size, an axillary node status, and a higher clinical stage were observed. Furthermore, they used a spontaneous breast cancer mouse strain with a triplenegative genotype to detect the sCLU dynamic expression in breast cancer oncogenesis. The sCLU mRNA and protein expression in the tumor and hyperplastic epithelium were upregulated and reached a peak compared with those of a normal mammary gland. These results suggest that sCLU is involved in the initiation of triple-negative breast cancer, such that a clinical trial of an anti-CLU treatment for triplenegative breast cancer would be beneficial. ${ }^{46}$

A previous study ${ }^{47}$ indicated that CLU expression was associated with estrogen and progesterone receptor-negative status and with the progression from primary carcinoma to metastatic carcinoma in lymph nodes. The study also described correlation between CLU expression and tumor size.

In a Phase II trial concluded in 2009 of custirsen in combination with docetaxel in metastatic breast cancer, custirsen was well tolerated and clinical activity was recorded, but the 
number of responses was insufficient to meet the criteria set for accrual of a second stage. ${ }^{48}$ The 15 patients included in this study were all positive for estrogen receptors, progesterone receptors, or both. No triple negatives were recorded. This research had very few patients and did not include triple-negative cases, so according to our view the results may be biased. In another study, clusterin inhibition in vivo and in vitro enhanced paclitaxel chemosensitivity in breast cancer cells. $^{49}$

\section{CLU and colorectal cancer}

Inhibition of thrombospondin-1 and clusterin abolished p38 mitogen-activated protein kinase phosphorylation, MMP-9 activity, and platelet-stimulated colon cancer invasion. Platelet-secreted thrombospondin-1 and clusterin promote the signal regulation of MMP-9 in platelet-induced colonic cancer invasion via a p38 mitogen-activated protein kinase-regulated pathway. These findings are relevant to the development of therapeutic approaches preventing and reducing tumor cell metastasis induced by colon adenocarcinoma. ${ }^{50}$

In the colon, overexpression of the sCLU was found only in the cytoplasm of highly infiltrating tumors and metastatic nodes. The increased level of the secreted form and the disappearance of the nuclear unglycosylated isoform were directly connected to increased cell survival, aggressiveness, and enhanced metastatic potential. ${ }^{10}$

Normal colonic mucosal cells depend on CLU for survival when a stressor condition appears, such as butyrateinduced cell death. ${ }^{51}$

\section{CLU and ovarian cancer}

Increased clusterin expression is correlated with more aggressive biologic behavior and impaired survival in ovarian cancer. ${ }^{52}$ Promoter methylation of the $C L U$ gene can be readily detected, although at low frequencies, in ovarian epi-

Table 2 Summary of findings in renal cell carcinoma

\begin{tabular}{ll}
\hline Reference & Results \\
\hline 65 & $\begin{array}{l}\text { Introducing the CLU gene into human renal cell } \\
\text { carcinoma enhances their metastatic potential } \\
\text { CLU inhibition using custirsen synergistically } \\
\text { enhanced antitumor activity of sorafenib }\end{array}$ \\
33 & $\begin{array}{l}\text { Suppression of CLU expression enhanced cisplatin- } \\
\text { induced cytotoxicity on renal cell carcinoma cells }\end{array}$ \\
32 & $\begin{array}{l}\text { Knockdown of CLU inhibits the growth and migration } \\
\text { of renal carcinoma cells }\end{array}$ \\
\hline
\end{tabular}

Abbreviation: CLU, clusterin. thelial tumors and is significantly associated with decreased protein expression of the gene..$^{53}$

Cyclic adenosine monophosphate (cAMP) induces apoptosis in granulosa cells of rat and human ovary by unidentified mechanisms. The apoptotic effect of cAMP was accompanied by an increase in the expression of $\mathrm{p} 53$ and Bax proteins. No change in Bcl-2 protein level was observed in cAMPtreated granulosa cells as compared to controls. These data suggest that cAMP may activate apoptosis in granulosa cells by shifting the ratio of the death promoter genes to death repressor genes via alteration of p53 and Bax expression. cAMP was also shown to inhibit CLU gene expression, suggesting a role for this protein in cAMP-induced apoptosis in granulosa cells. ${ }^{54}$

Treatment of granulosa cells with an antisense oligonucleotide against CLU resulted in an increase in the apoptotic cell death compared to the control. These findings indicate that depletion of CLU can lead to the programmed cell death in the ovary, suggesting a functional role for this protein in follicular atresia. ${ }^{55}$

\section{CLU and pancreatic cancer}

CLU induction accompanies pancreas regeneration in the rat. ${ }^{56} \mathrm{CLU}$ induction was accompanied in the regenerating pancreas by a robust development of new lobules with ductules, acini, and endocrine islets after partial pancreatectomy. In $C L U$ knockout mice, however, pancreatectomy resulted in a poor formation of regenerating lobules. In particular, regeneration of beta cells was also significantly reduced and was associated with persistent hyperglycemia. Duct cells obtained from pancreatectomized CLU knockout mice exhibited impaired beta-cell formation in vitro; this was restored by administration of exogenous CLU. These findings suggest that CLU plays a critical role to promote both exocrine and endocrine regeneration following pancreatic injury, as well as for in vitro beta-cell regeneration, so that CLU is an important factor in pancreatic growth.

Transfection of clusterin cDNA into primary cultured pancreatic duct cells resulted in a 2.5-fold increase in cell proliferation and induced transformation of nondifferentiated duct cells into differentiated cells displaying cytokeratin immunoreactivity. These results confirm that CLU may play essential roles in the neogenic regeneration of pancreatic tissue by stimulating proliferation and differentiation of duct cells. ${ }^{57}$

Knockdown of $C L U$ sensitizes pancreatic cancer cells to gemcitabine chemotherapy by ERK1/2 inactivation. ${ }^{58,59}$ 


\section{CLU and hepatocarcinoma}

Hepatocellular carcinoma (HCC) is one of the most common and aggressive tumors. The prognosis of $\mathrm{HCC}$ is extremely poor with a five-year survival rate of less than $5 \%$ for late primary HCC. Despite recent advances in the diagnosis and treatment of $\mathrm{HCC}$, the mortality rate of $\mathrm{HCC}$ remains high and is mainly attributed to the high rate of intrahepatic metastasis after treatment. ${ }^{60}$

High levels of sCLU expression have been associated with migration, invasion, and metastasis, including HCC. ${ }^{61}$ It has been shown that CLU is overexpressed in metastatic human HCC tissue compared to nonmetastatic HCC tissue, ${ }^{61}$ implicating a role for CLU in HCC progression.

Recent studies showed that downregulation of CLU sensitizes cells to chemotherapy and radiotherapy, not only in prostate cancer but also in other malignant tissues like the lung and breast. ${ }^{62,45}$ In hepatocellular carcinoma, it was not clear whether $C L U$ silencing inhibited the invasion and metastasis, but Chen et a $l^{60}$ provided evidence that CLU plays an important role in HCC invasiveness by increasing MMP-2 expression and decreasing E-cadherin expression.

Lau et $a{ }^{61}$ showed that overexpression of CLU in hepatoma cells transfected with $C L U$ genes increased cell migration by twofold in vitro and formation of metastatic tumor nodules in the liver by eightfold in vivo. sCLU overexpression contributes to oxaliplatin resistance by activating the AKT pathway in hepatocellular carcinoma ${ }^{64}$

\section{CLU in renal cell carcinoma}

Introducing the $C L U$ gene into human CCRC enhances the metastatic potential (Table 2). ${ }^{65}$ We mentioned previously that CLU inhibition using custirsen synergistically enhanced the antitumor activity of sorafenib in a human CCRC model..$^{33}$

\section{CLU and endometrial and cervical cancer}

Estrogen increases the paclitaxel resistance of endometrial cancer cell lines by increasing CLU expression. ${ }^{66} \mathrm{CLU}$ confers paclitaxel resistance in cervical cancer. ${ }^{67}$

\section{CLU and lung cancer}

Yan et $a^{168}$ suggested that sCLU plays a positive role in nonsmall-cell lung cancer (NSCLC) cell proliferation, which may be mediated by the PI3K/AKT signaling pathway, and demonstrated that RNA interference-mediated $s C L U$ gene silencing may provide a novel therapeutic strategy in the treatment of NSCLC because it reduced tumor cell proliferation, blocked the PI3K/AKT pathway, and induced apoptosis.
Although CLU expression has been associated with various human malignancies, the mechanisms by which CLU promotes cancer progression and metastasis have not been fully elucidated. Chou et $a l^{63}$ found that CLU regulates epithelial-to-mesenchymal transitions and aggressive behavior of human lung adenocarcinoma cells through modulating ERK signaling and Slug/E-cadherin expression.

In small cell lung cancer cell lines, oblimersen, an antisense Bcl-2 oligodeoxynucleotide, has increased response to radiation in vivo and in vitro. The mechanism of action of oblimersen resembles that of custirsen in the sense of increasing apoptosis. ${ }^{69}$

\section{CLU and melanoma}

Hoeller et $\mathrm{al}^{70}$ reported that in tissue samples, CLU expression was low in nevi but high in primary melanoma and melanoma metastases. CLU was also strongly expressed in melanoma cell lines but was barely detectable in cultured melanocytes. To elucidate a possible role of CLU in melanoma drug resistance, CLU expression was regulated by either plasmid-driven overexpression or by antisense-mediated downregulation. CLU overexpression was associated with an increase in drug resistance, ie, with an increased survival of melanoma cells in the presence of cytotoxic drugs. In contrast, downregulation of CLU by antisense oligonucleotides directed against CLU mRNA significantly reduced drug resistance.

Shannan et $\mathrm{l}^{71}$ studied the expression of CLU immunohistochemically in primary cutaneous malignant melanomas, metastasis of melanomas, and acquired melanocytic nevi. CLU immunoreactivity was found in the two former cases in contrast to acquired nevi. Treatment with vitamin D was observed to modulate CLU expression in vitamin-Dresponsive cells but not in resistant melanoma cell lines.

\section{CLU and head and neck squamous cell carcinoma}

Overexpression of CLU in head and neck squamous cell carcinoma has been reported, but no relationship implications were ade. ${ }^{72}$

\section{Early versus late inhibition of CLU}

There are certain evidences that inhibition of sCLU in late stages of tumor evolution may have beneficial effects in therapy, whereas in the early stages, effects may be the opposite.

In vitro, $C L U$ silencing by antisense oligonucleotides and siRNAs directed against CLU mRNA in CLU-rich lung cancer cell lines sensitized cells to chemotherapy and radiotherapy and decreased their metastatic potential. In vivo, a recent work analyzed the prognostic role of CLU in NSCLC, 
showing that CLU-positive patients with lung cancer had a better overall survival and disease-free survival than those with CLU-negative tumors. ${ }^{62}$ These data are contradictory to the promising in vitro results.

Based on this, Panico et $\mathrm{al}^{62}$ theorize that in earlystage lung cancers CLU represents a positive biomarker correlating with better overall survival. In patients with advanced cancer, already treated with chemotherapy and radiotherapy, the induction of CLU may confer resistance to the treatments.

\section{Summary and future perspectives}

The targeting of the apoptotic pathway as a new cancer treatment modality is in accelerated development with novel drugs like oblimersen (a Bcl-2 antisense oligonucleotide blocker), ${ }^{69,72,73}$ and a combination of apatorsen (OGX-427, an antisense oligonucleotide that targets HSP27; OncoGeneX Pharmaceuticals Inc.), gemcitabine, and cisplatin is currently being investigated in a Phase II trial of patients with advanced bladder cancer. $^{74}$

CLU has been identified as a valid target for cancer treatment, not only because of its antiapoptotic actions after chemotherapy, but also for other protumor effects it can produce, like PI3K/AKT pathway activation, vascular endothelial growth factor increase, ERK1/2 signaling, MMP-9 overexpression, and $\mathrm{NF}-\kappa \mathrm{B}$ regulation. CLU inhibition using custirsen synergistically enhances heat-shock protein 90 inhibitor activity by suppressing the heat-shock response in castrate-resistant prostate cancer. ${ }^{75}$

The possible future of custirsen, once it is approved by the US Food and Drug Administration for castration-resistant prostate cancer with metastasis, will be an extension of indications to more tumors. Preclinical evidence warrants the testing of custirsen in CCRC, breast cancer (specifically in triple-negative cases), pancreatic adenocarcinoma, and hepatocarcinoma. In the case of breast cancer, the work by Zhang et $\mathrm{al}^{46}$ and Redondo et $\mathrm{al}^{47}$ warrants further research of custirsen in spite of the lack of evidence of clinical benefit in the research by Chia et al. ${ }^{48}$

There are certain clues that indicate that inhibition of CLU is a valid therapy in advanced cancer, but this may not be true in early cases. ${ }^{62}$ Thus, a major issue in cancer evolution that remains unanswered is if CLU represents a driving force of tumorigenesis or a late phenomenon after chemotherapy. According to the literature here reviewed, we are inclined to think of clusterin as a protein that is overexpressed after intensive chemotherapy and plays a key role in the antiapoptotic defenses against cellular stress induced by chemotherapy. Consequently, CLU inhibition should probably not be attempted in early-stage treatment of cancer.

\section{Disclosure}

The author reports no conflicts of interest in this work.

\section{References}

1. Bettuzzi S, Hiipakka RA, Gilna P, Liao ST. Identification of an androgen-repressed mRNA in rat ventral prostate as coding for sulphated glycoprotein 2 by cDNA cloning and sequence analysis. Biochem $J$. 1989;257(1):293-296.

2. Sensibar JA, Sutkowski DM, Raffo A, et al. Prevention of cell death induced by tumor necrosis factor alpha in LNCaP cells by overexpression of sulfated glycoprotein-2 (clusterin). Cancer Res. 1995;55(11): 2431-2437.

3. Trougakos IP, So A, Jansen B, Gleave ME, Gonos ES. Silencing expression of the clusterin/apolipoprotein $\mathrm{j}$ gene in human cancer cells using small interfering RNA induces spontaneous apoptosis, reduced growth ability, and cell sensitization to genotoxic and oxidative stress. Cancer Res. 2004;64(5):1834-1842.

4. Aronow BJ, Lund SD, Brown TL, Harmony JA, Witte DP. Apolipoprotein J expression at fluid-tissue interfaces: potential role in barrier cytoprotection. Proc Natl Acad Sci U S A. 1993;90(2):725-729.

5. Michel D, Chatelain G, North S, Brun G. Stress-induced transcription of the clusterin/apoJ gene. Biochem J. 1997;328 (Pt 1):45-50.

6. Rizzi F, Bettuzzi S. The clusterin paradigm in prostate and breast carcinogenesis. Endocr Relat Cancer. 2010;17(1):R1-R17.

7. Leskov KS, Klokov DY, Li J, Kinsella TJ, Boothman DA. Synthesis and functional analyses of nuclear clusterin, a cell death protein. J Biol Chem. 2003;278(13):11590-11600.

8. Essabbani A, Garcia L, Zonetti MJ, Fisco T, Pucci S, Chiocchia G. Exon-skipping strategy by ratio modulation between cytoprotective versus pro-apoptotic clusterin forms increased sensitivity of $\mathrm{LNCaP}$ to cell death. PLoS One. 2013;8(2):e54920.

9. Trougakos IP, Djeu JY, Gonos ES, Boothman DA. Advances and challenges in basic and translational research on clusterin. Cancer Res. 2009;69(2):403-406.

10. Pucci S, Bonanno E, Pichiorri F, Angeloni C, Spagnoli LG. Modulation of different clusterin isoforms in human colon tumorigenesis. Oncogene. 2004;23(13):2298-2304.

11. Cochrane DR, Wang Z, Muramaki M, Gleave ME, Nelson CC. Differential regulation of clusterin and its isoforms by androgens in prostate cells. J Biol Chem. 2007;282(4):2278-2287.

12. Loison F, Debure L, Nizard P, le Goff P, Michel D, le Dréan Y. Up-regulation of the clusterin gene after proteotoxic stress: implication of HSF 1-HSF2 heterocomplexes. Biochem J. 2006;395(1): 223-231.

13. Viard I, Wehrli P, Jornot L, et al. Clusterin gene expression mediates resistance to apoptotic cell death induced by heat shock and oxidative stress. J Invest Dermatol. 1999;112(3):290-296.

14. Trougakos IP, Lourda M, Antonelou MH, et al. Intracellular clusterin inhibits mitochondrial apoptosis by suppressing p53-activating stress signals and stabilizing the cytosolic Ku70-Bax protein complex. Clin Cancer Res. 2009;15(1):48-59.

15. Ammar H, Closset JL. Clusterin activates survival through the phosphatidylinositol 3-kinase/Akt pathway. J Biol Chem. 2008;283(19): 12851-12861.

16. Ma X, Bai Y. IGF-1 activates the P13K/AKT signaling pathway via upregulation of secretory clusterin. Mol Med Rep. 2012;6(6): 1433-1437.

17. Wang X, Luo L, Dong D, Yu Q, Zhao K. Clusterin plays an important role in clear renal cell cancer metastasis. Urol Int. Epub August 29, 2013. 
18. FuY, Lai Y, Wang Q, et al. Overexpression of clusterin promotes angiogenesis via the vascular endothelial growth factor in primary ovarian cancer. Mol Med Rep. 2013;7(6):1726-1732.

19. Wei L, Xue T, Wang J, et al. Roles of clusterin in progression, chemoresistance and metastasis of human ovarian cancer. Int J Cancer. 2009;125(4):791-806.

20. Essabbani A, Margottin-Goguet F, Chiocchia G. Identification of clusterin domain involved in NF-kappaB pathway regulation. $J$ Biol Chem. 2010;285(7):4273-4277.

21. Zoubeidi A, Ettinger S, Beraldi E, et al. Clusterin facilitates COMMD1 and I-kappaB degradation to enhance NF-kappaB activity in prostate cancer cells. Mol Cancer Res. 2010;8(1):119-130.

22. Serrano A, Redondo M, Tellez T, et al. Regulation of clusterin expression in human cancer via DNA methylation. Tumour Biol. 2009;30(5-6):286-291.

23. Wang C, Jiang K, Gao D, et al. Clusterin protects hepatocellular carcinoma cells from endoplasmic reticulum stress induced apoptosis through GRP78. PLoS One. 2013;8(2):e55981.

24. Zoubeidi A, Chi K, Gleave M. Targeting the cytoprotective chaperone, clusterin, for treatment of advanced cancer. Clin Cancer Res. 2010;16(4):1088-1093.

25. Park DC, Yeo SG, Wilson MR, et al. Clusterin interacts with paclitaxel and confer paclitaxel resistance in ovarian cancer. Neoplasia. 2008;10(9):964-972.

26. Tang M, Li J, Liu B, Song N, Wang Z, Yin C. Clusterin expression and human testicular seminoma. Med Hypotheses. 2013;81(4):635-637.

27. Zielinski RR, Eigl BJ, Chi KN. Targeting the apoptosis pathway in prostate cancer. Cancer J. 2013;19(1):79-89.

28. Goetz EM, Shankar B, Zou Y, et al. ATM-dependent IGF-1 induction regulates secretory clusterin expression after DNA damage and in genetic instability. Oncogene. 2011;30(35):3745-3754.

29. Klock G, Storch S, Rickert J, Gutacker C, Koch-Brandt C. Differential regulation of the clusterin gene by Ha-ras and c-myc oncogenes and during apoptosis. J Cell Physiol. 1998;177(4):593-605.

30. Criswell T, Klokov D, Beman M, Lavik JP, Boothman DA. Repression of IR-inducible clusterin expression by the p53 tumor suppressor protein. Cancer Biol Ther. 2003;2(4):372-380.

31. Higano CS. Potential use of custirsen to treat prostate cancer. Onco Targets Ther. 2013;6:785-797.

32. Shi H, Deng JH, Wang Z, Cao KY, Zhou L, Wan H. Knockdown of clusterin inhibits the growth and migration of renal carcinoma cells and leads to differential gene expression. Mol Med Rep. 2013;8(1):35-40.

33. Kususda Y, Miyake H, Gleave ME, Fujisawa M. Clusterin inhibition using OGX-011 synergistically enhances antitumour activity of sorafenib in a human renal cell carcinoma model. Br J Cancer. 2012;106(12):1945-1952.

34. Lee CH, Jin RJ, Kwak C, et al. Suppression of clusterin expression enhanced cisplatin-induced cytotoxicity on renal cell carcinoma cells. Urology. 2002;60(3):516-520.

35. Niu ZH, Wang Y, Chun B, Li CX, Wu L. Secretory clusterin (sCLU) overexpression is associated with resistance to preoperative neoadjuvant chemotherapy in primary breast cancer. Eur Rev Med Pharmacol Sci. 2013;17(10):1337-1344.

36. Niu Z, Li X, Hu B, et al. Small interfering RNA targeted to secretory clusterin blocks tumor growth, motility, and invasion in breast cancer. Acta Biochim Biophys Sin (Shanghai). 2012;44(12):991-998.

37. Li J, Jia L, Zhao P, Jiang Y, Zhong S, Chen D. Stable knockdown of clusterin by vectorbased RNA interference in a human breast cancer cell line inhibits tumour cell invasion and metastasis. J Int Med Res. 2012;40(2):545-555.

38. Flanagan L, Whyte L, Chatterjee N, Tenniswood M. Effects of clusterin over-expression on metastatic progression and therapy in breast cancer. BMC Cancer. 2010;10:107.

39. Laskin JJ, Nicholas G, Lee C, et al. Phase I/II trial of custirsen (OGX-011), an inhibitor of clusterin, in combination with a gemcitabine and platinum regimen in patients with previously untreated advanced non-small cell lung cancer. J Thorac Oncol. 2012;7(3):579-586.
40. Lubin J, Markowska A, Knapp P. Factors affecting response of chemotherapy in women with ovarian cancer. Eur J Gynaecol Oncol. 2012;33(6):644-647.

41. Miyake H, Hara S, ZellwegerT, Kamidono S, Gleave ME, Hara I. Acquisition of resistance to Fas-mediated apoptosis by overexpression of clusterin in human renal-cell carcinoma cells. Mol Urol. 2001;5(3): 105-111.

42. Zellweger T, Chi K, Miyake H, et al. Enhanced radiation sensitivity in prostate cancer by inhibition of the cell survival protein clusterin. Clin Cancer Res. 2002;8(10):3276-3284.

43. Gleave ME, Miyake H, Zellweger T, et al. Use of antisense oligonucleotides targeting the antiapoptotic gene, clusterin/testosterone-repressed prostate message 2 , to enhance androgen sensitivity and chemosensitivity in prostate cancer. Urology. 2001;58(2 Suppl 1): 39-49.

44. Hassan MK, Watari H, Han Y, et al. Clusterin is a potential molecular predictor for ovarian cancer patient's survival: targeting clusterin improves response to paclitaxel. J Exp Clin Cancer Res. 2011;30:113.

45. Wang Y, Wang X, Zhao H, Liang B, Du Q. Clusterin confers resistance to TNF-alpha-induced apoptosis in breast cancer cells through NF-kappaB activation and Bcl-2 overexpression. J Chemother. 2012;24(6):348-357.

46. Zhang D, Sun B, Zhao X, et al. Secreted CLU is associated with the initiation of triple-negative breast cancer. Cancer Biol Ther. 2012;13(5):321-329.

47. Redondo M, Villar E, Torres-Muñoz J, Tellez T, Morell M, Petito CK. Overexpression of clusterin in human breast carcinoma. Am J Pathol. 2000;157(2):393-399.

48. Chia S, Dent S, Ellard S, et al. Phase II trial of OGX-011 in combination with docetaxel in metastatic breast cancer. Clin Cancer Res. 2009;15(2):708-713.

49. So A, Sinnemann S, Huntsman D, Fazli L, Gleave M. Knockdown of the cytoprotective chaperone, clusterin, chemosensitizes human breast cancer cells both in vitro and in vivo. Mol Cancer Ther. 2005;4(12): 1837-1849.

50. Radziwon-Balicka A, Santos-Martinez MJ, Corbalan JJ, et al. Mechanisms of platelet-stimulated colon cancer invasion: role of clusterin and thrombospondin 1 in regulation of the P38MAPK-MMP-9 pathway. Carcinogenesis. Epub November 27, 2013.

51. Cohen L, Azriel-Tamir H, Arotsker N, Sekler I, Hershfinkel M. Zinc sensing receptor signaling, mediated by GPR39, reduces butyrateinduced cell death in HT29 colonocytes via upregulation of clusterin. PLoS One. 2012;7(4):e35482.

52. Yang GF, Li XM, Xie D. Overexpression of clusterin in ovarian cancer is correlated with impaired survival. Int J Gynecol Cancer. 2009;19(8): 1342-1346.

53. Yang G, Zhang H, Liu Y, et al. Epigenetic and immunohistochemical characterization of the Clusterin gene in ovarian tumors. Arch Gynecol Obstet. 2013;287(5):989-995.

54. Zwain IH, Amato P. cAMP-induced apoptosis in granulosa cells is associated with up-regulation of P53 and bax and down-regulation of clusterin. Endocr Res. 2001;27(1-2):233-249.

55. Zwain I, Amato P. Clusterin protects granulosa cells from apoptotic cell death during follicular atresia. Exp Cell Res. 2000;257(1): 101-110.

56. Lee $\mathrm{S}$, Hong $\mathrm{SW}$, Min BH, et al. Essential role of clusterin in pancreas regeneration. Dev Dyn. 2011;240(3):605-615.

57. Min BH, Kim BM, Lee SH, Kang SW, Bendayan M, Park IS. Clusterin expression in the early process of pancreas regeneration in the pancreatectomized rat. J Histochem Cytochem. 2003;51(10): 1355-1365.

58. Tang Y, Liu F, Zheng C, Sun S, Jiang Y. Knockdown of clusterin sensitizes pancreatic cancer cells to gemcitabine chemotherapy by ERK1/2 inactivation. J Exp Clin Cancer Res. 2012;31:73.

59. Chen Q, Wang Z, Zhang K, et al. Clusterin confers gemcitabine resistance in pancreatic cancer. World J Surg Oncol. 2011;9:59.

60. Chen D, Wang Y, Zhang K, Jiao X, Yan B, Liang J. Antisense oligonucleotide against clusterin regulates human hepatocellular carcinoma invasion through transcriptional regulation of matrix metalloproteinase-2 and e-cadherin. Int J Mol Sci. 2012;13(8):10594-10607. 
61. Lau SH, Sham JS, Xie D, et al. Clusterin plays an important role in hepatocellular carcinoma metastasis. Oncogene. 2006;25(8): 1242-1250.

62. Panico F, Rizzi F, Fabbri LM, Bettuzzi S, Luppi F. Clusterin (CLU) and lung cancer. Adv Cancer Res. 2009;105:63-76.

63. Chou TY, Chen WC, Lee AC, Hung SM, Shih NY, Chen MY. Clusterin silencing in human lung adenocarcinoma cells induces a mesenchymalto-epithelial transition through modulating the ERK/Slug pathway. Cell Signal. 2009;21(5):704-711.

64. Xiu P, Dong X, Dong X, et al. Secretory clusterin contributes to oxaliplatin resistance by activating Akt pathway in hepatocellular carcinoma. Cancer Sci. 2013;104(3):375-382.

65. Miyake H, Gleave ME, Arakawa S, Kamidono S, Hara I. Introducing the clusterin gene into human renal cell carcinoma cells enhances their metastatic potential. J Urol. 2002;167(5):2203-2208.

66. Won YS, Lee SJ, Yeo SG, Park DC. Effects of female sex hormones on clusterin expression and paclitaxel resistance in endometrial cancer cell lines. Int J Med Sci. 2012; 9(1):86-92.

67. Park DC, Yeo SG, Shin EY, Mok SC, Kim DH. Clusterin confers paclitaxel resistance in cervical cancer. Gynecol Oncol. 2006;103(3):996-1000.

68. Yan Y, Luo K, Zhang H, Chai W. RNA interference-mediated secretory clusterin gene silencing inhibits proliferation and promotes apoptosis of human non-small cell lung cancer cells. Hepatogastroenterology. 2013;60(121):70-75.

69. Loriot Y, Mordant P, Brown BD, Bourhis J, Soria JC, Deutsch E. Inhibition of BCL-2 in small cell lung cancer cell lines with oblimersen, an antisense BCL-2 oligodeoxynucleotide (ODN): in vitro and in vivo enhancement of radiation response. Anticancer Res. 2010;30(10): 3869-3878.
70. Hoeller C, Pratscher B, Thallinger C, et al. Clusterin regulates drug-resistance in melanoma cells. J Invest Dermatol. 2005;124(6): 1300-1307.

71. Shannan B, Seifert M, Leskov K, et al. Clusterin (CLU) and melanoma growth: CLU is expressed in malignant melanoma and 1,25dihydroxyvitamin D3 modulates expression of CLU in melanoma cell lines in vitro. Anticancer Res. 2006;26(4A):2707-2716.

72. Bijian K, Mlynarek AM, Balys RL, et al. Serum proteomic approach for the identification of serum biomarkers contributed by oral squamous cell carcinoma and host tissue microenvironment. J Proteome Res. 2009;8(5):2173-2185.

73. Advani PP, Paulus A, Masood A, Sher T, Chanan-Khan A. Pharmacokinetic evaluation of oblimersen sodium for the treatment of chronic lymphocytic leukemia. Expert Opin Drug Metab Toxicol. 2011;7(6):765-774.

74. Ischia J, So AI. The role of heat shock proteins in bladder cancer. Nat Rev Urol. 2013;10(7):386-395.

75. Lamoureux F, Thomas C, Yin MJ, et al. Clusterin inhibition using OGX-011 synergistically enhances Hsp90 inhibitor activity by suppressing the heat shock response in castrate-resistant prostate cancer. Cancer Res. 2011;71(17):5838-5849.
OncoTargets and Therapy

\section{Publish your work in this journal}

OncoTargets and Therapy is an international, peer-reviewed, open access journal focusing on the pathological basis of all cancers, potential targets for therapy and treatment protocols employed to improve the management of cancer patients. The journal also focuses on the impact of management programs and new therapeutic agents and protocols on

\section{Dovepress}

patient perspectives such as quality of life, adherence and satisfaction The manuscript management system is completely online and includes a very quick and fair peer-review system, which is all easy to use. Visit http://www.dovepress.com/testimonials.php to read real quotes from published authors. 